\title{
Activated amelogenin Y-linked (AMELY) regulation and angiogenesis in human hepatocellular carcinoma by biocomputation
}

\author{
LIANXIU QI $^{1 *}$, LIN WANG $^{1,3 *}$, JUXIANG HUANG $^{1 *}$, MINGHU JIANG $^{2 *}$, HAIZHEN DIAO $^{1}$, \\ HUILEI ZHOU ${ }^{1}$, XIAOHE $\mathrm{LI}^{1}$ and ZHENFU JIANG ${ }^{3}$
}

\begin{abstract}
${ }^{1}$ Biomedical Center, School of Electronic Engineering, Beijing University of Posts and Telecommunications, Beijing 100876;
${ }^{2}$ Lab of Computational Linguistics, School of Humanities and Social Sciences, Tsinghua University, Beijing 100084;

${ }^{3}$ Shandong Longkou Yimin Molecular Drug Network Research and Development Center, Shandong 265700, P.R. China
\end{abstract}

Received October 14, 2012; Accepted January 7, 2013

DOI: 10.3892/ol.2013.1122

\begin{abstract}
In the present study, a comparison of the biological processes and gene ontology (GO) in human hepatocellular carcinoma (HCC) with high expression (fold change $\geq 2$ ) of amelogenin Y-linked (AMELY)-activated upstream regulation networks with non-tumor hepatitis/cirrhotic tissues (HBV or HCV infection) with low expression of activated networks was performed. The principle biological processes involved in non-tumor hepatitis/cirrhotic tissues include positive regulation of mismatch repair, regulation of transcription from RNA polymerase II promoters, negative regulation of cell-cell adhesion, protein ubiquitinatin and protein catabolism. The main biological processes involved in the development of HCC include positive regulation of calcium ion transport into the cytosol, cell proliferation, DNA replication, fibroblast proliferation, immune response, microtubule polymerization and protein secretion. Specific transcription from RNA polymerase II promoters, regulation of angiogenesis, cell growth, protein metabolism, Wnt receptor signaling pathways, negative regulation of endothelial cell differentiation, microtubule depolymerization, peptidase activity and progression through the cell cycle are also involved. Positive regulation of transcription is involved in both processes. An activated AMELY-coupled upstream positive regulation of immune response-mediated
\end{abstract}

Correspondence to: Professor Lin Wang, Biomedical Center, School of Electronic Engineering, Beijing University of Posts and Telecommunications, 10 Xitucheng road, Haidian, Beijing 100876, P.R. China

E-mail: wanglin98@tsinghua.org.cn

*Contributed equally

Key words: activated amelogenin Y-linked (AMELY) upstream regulation network, Wnt signaling, calcium signaling, cell growth, angiogenesis, theoretical analysis, hepatocellular carcinoma protein secretion to Wnt signaling and calcium into cytosolinduced regulation of cell growth and angiogenesis in HCC is proposed. The AMELY upstream regulation molecular network model was constructed with $B U B 1 B, C S T 6, E S M 1$, HOXA5, LEF1, MAPT, MYBL2, NOTCH3, PLA2G1B, $P R O K 1, R O B O 1, S C M L 2$ and $U B E 2 C$ in HCC from a Gene Expression Omnibus (GEO) dataset by gene regulation network inference methods and our programming methods.

\section{Introduction}

Amelogenin Y-linked (AMELY) has been identified to be one of the significantly highly expressed (fold change $\geq 2$ ) genes in human hepatocellular carcinoma (HCC). Systemscomputational analysis was used to elucidate the function and mechanism of $A M E L Y$-activated upstream regulation networks in HCC compared with low or non-tumor hepatitis/ cirrhotic tissues (HBV or HCV infection) from GEO dataset GSE10140-10141 (1).

The possible correlations among immune response, protein secretion, Wnt signaling and calcium release into the cytosol with cancer or tumor formation have been investigated in several studies. Examples include the tetraspanins and the immune response against cancer (2); induction of tumor-specific immune response by gene transfer of Hsp70 cell-penetrating peptide fusion protein and tumors in mice (3); in situ immune responses after neoadjuvant chemotherapy for breast cancer predicting survival (4); human tumor cells killed by anthracyclines inducing a tumor-specific immune response (5); keratin 17 promoting epithelial proliferation and tumor growth by polarizing the immune response in skin (6); interleukin-10, but not interleukin-18, possibly being associated with the immune response against well-differentiated thyroid cancer (7); CXCR7 protein expression correlating with elevated mmp-3 secretion in breast cancer cells (8); heat shock cognate 70 protein secretion as a new growth arrest signal for cancer cells (9); overexpression of p53 protein and local hGH, IGF-I, IGFBP-3, IGFBP-2 and PRL secretion by human breast cancer explants (10); restoration of full-length APC protein in SW480 colon cancer cells inducing exosome- 
mediated secretion of DKK-4 (11); cancer cell secretion of the DAMP protein HMGB1 supporting progression in malignant mesothelioma (12); targeting Wnt signaling in colon cancer stem cells (13); protein cross-talk in CD133+ colon cancer cells indicating activation of the Wnt pathway and upregulation of SRp20 that is potentially involved in tumorigenicity (14); miRNA-34 intrinsically linking p53 tumor suppressor and Wnt signaling (15); the Wnt/ $/$-catenin pathway regulating self-renewal of cancer stem-like cells in human gastric cancer (16); a serrated colorectal cancer pathway predominating over the classic Wnt pathway in patients with hyperplastic polyposis syndrome (17); insights from studies with oral cleft genes suggesting associations between Wnt pathway genes and risk of oral cancer (18); and hydrogen sulfide increasing calcium-activated potassium (BK) channel activity of rat pituitary tumor cells (19). The function and mechanism of the high expression of the $A M E L Y$-activated upstream regulation network in HCC is not known and remains to be elucidated.

The aim of this study was to compare the biological processes and occurrence numbers of gene ontology (GO) in non-tumor hepatitis/cirrhotic tissues (HBV or HCV infection) with low expression of $A M E L Y$ upstream regulation networks and the corresponding HCC tissue with high expression (fold change $\geq 2$ ) of $A M E L Y$. AMELY-activated upstream regulation molecular networks in non-tumor hepatitis/cirrhotic tissues and HCC were constructed. A further aim was to identify the $A M E L Y$ upstream regulation molecular network involved in HCC.

\section{Materials and methods}

Materials. Microarrays from of 6,144 genes from 25 non-tumor hepatitis/cirrhotic tissues and 25 HCC patients were used for analyzing the possible AMELY-activated upstream regulation networks of HCC based on GEO dataset GSE10140-10141 (http://www.ncbi.nlm.nih.gov/geo/query/ acc.cgi?acc=GSE10140 and http://www.ncbi.nlm.nih.gov/geo/ query/acc.cgi?acc=GSE10141). The raw microarray data were pre-processed using log base 2 .

Methods. A total of 225 molecules with a significantly high expression (fold change $\geq 2$ ) in HCC were identified for studying the function and mechanism of $A M E L Y$-activated upstream regulation networks in $\mathrm{HCC}$ by systems-computational analysis of difference with low or non-tumor hepatitis/cirrhotic tissues using significant analysis of microarrays (SAM; http:// www-stat.stanford.edu/ tibs/SAM/) (20). We selected the two-class paired data and a minimum fold change $\geq 2$ under the false-discovery rate of $0 \%$.

The $A M E L Y$-activated upstream regulation network of HCC was analyzed using the Molecule Annotation System, MAS (CapitalBio Corporation, Beijing, China). The primary databases of MAS integrated various well-known biological resources, such as Gene Ontology (http://www.geneontology. org), KEGG (http://www.genome.jp/kegg/), BioCarta (http:// www.biocarta.com/), GenMapp (http://www.genmapp. org/), HPRD (http://www.hprd.org/), MINT (http://mint.bio. uniroma2.it/mint/Welcome.do), BIND (http://www.blueprint. org/), Intact (http://www.ebi.ac.uk/intact/), UniGene (www.
Table I. Biological processes of AMELY upstream regulation network in non-tumor hepatitis/cirrhotic tissues using our programming.

Biological process

Occurrence of GO term

Negative regulation of cell-cell adhesion 1

Negative regulation of protein catabolism

Negative regulation of protein ubiquitination

1

Positive regulation of mismatch repair

Positive regulation of transcription

1

1

Regulation of transcription from

RNA polymerase II promoter

1

GO, gene ontology; AMELY, amelogenin Y-linked.



Figure 1. Amelogenin Y-linked (AMELY)-activated upstream regulation molecular network in non tumor hepatitis/cirrhotic tissues by GRNInfer and our programming.

ncbi.nlm.nih.gov/UniGene) and OMIM (http://www.ncbi. nlm.nih.gov/entrez/query.fcgi?db=OMIM).

Biological processes and occurrence numbers of $\mathrm{GO}$ in non-tumor hepatitis/cirrhotic tissues (HBV or HCV infection) with activated low expression of AMELY upstream regulation networks and the corresponding HCC tissues with high expression (fold change $\geq 2$ ) were identified and computed.

$A M E L Y$-activated upstream regulation molecular networks in non-tumor hepatitis/cirrhotic tissues and HCC were constructed by GRNInfer (21) and published studies (22-36), and illustrated by GVedit tool, respectively.

\section{Results}

The biological processes of AMELY-activated upstream regulation networks in non-tumor hepatitis/cirrhotic tissues and HCC are presented in Tables I and II, respectively. The $A M E L Y$-activated upstream regulation molecular network consisted of $C A D, C E B P A, M Y C N$ and $P R K C G$ in non-tumor hepatitis/cirrhotic tissues, as shown in Fig. 1. The AMELYactivated upstream regulation molecular network included BUB1B, CST6, ESM1, HOXA5, LEF1, MAPT, MYBL2, NOTCH3, PLA2G1B, PROK1, ROBO1, SCML2 and UBE2C in HCC, as shown in Fig. 2. 
Table II. Biological processes of AMELY upstream regulation network in human $\mathrm{HCC}$ using our programming.

\begin{tabular}{cc} 
Biological process & $\begin{array}{c}\text { Occurrence of } \\
\text { GO term }\end{array}$ \\
\hline
\end{tabular}

Negative regulation of endothelial cell

differentiation

Negative regulation of microtubule

depolymerization

Negative regulation of peptidase activity

Negative regulation of progression through

cell cycle

Positive regulation of calcium ion transport

into cytosol

Positive regulation of cell proliferation

Positive regulation of DNA replication

Positive regulation of fibroblast proliferation

1

Positive regulation of immune response

\section{1}

Positive regulation of microtubule

polymerization

Positive regulation of protein secretion

Positive regulation of specific transcription

from RNA polymerase II promoter

1

Positive regulation of transcription

Regulation of angiogenesis

Regulation of cell growth

Regulation of protein metabolism

Regulation of Wnt receptor signaling pathway

GO, gene ontology; AMELY, amelogenin Y-linked; HCC, hepatocellular carcinoma.

\section{Discussion}

The aim of this study was to elucidate the function and mechanism of $A M E L Y$-activated upstream regulation networks in HCC using systems-computational analysis of differences and similarities with non-tumor hepatitis/cirrhotic tissues (HBV or HCV infection). Biological processes and occurrence numbers of GO in non-tumor tissues with activated low expression of $A M E L Y$ upstream regulation networks and the corresponding HCC tissues with high expression (fold change $\geq 2$ ) were identified and computed (Tables I and II).

We analyzed and compared the biological processes and occurrence numbers of GO in HCC tissues with high expression (fold change $\geq 2$ ) of the $A M E L Y$-activated upstream regulation network and the corresponding non-tumor hepatitis/cirrhotic tissues with low expression of the activated network. The biological processes identified solely in non-tumor hepatitis/ cirrhotic tissues included negative regulation of cell-cell adhesion, transcription from RNA polymerase II promoter, protein ubiquitination and protein catabolism, as well as positive regulation of mismatch repair. The processes found only in HCC consisted of negative regulation of endothelial cell differentiation, microtubule depolymerization, peptidase activity, progression through the cell cycle, positive regulation

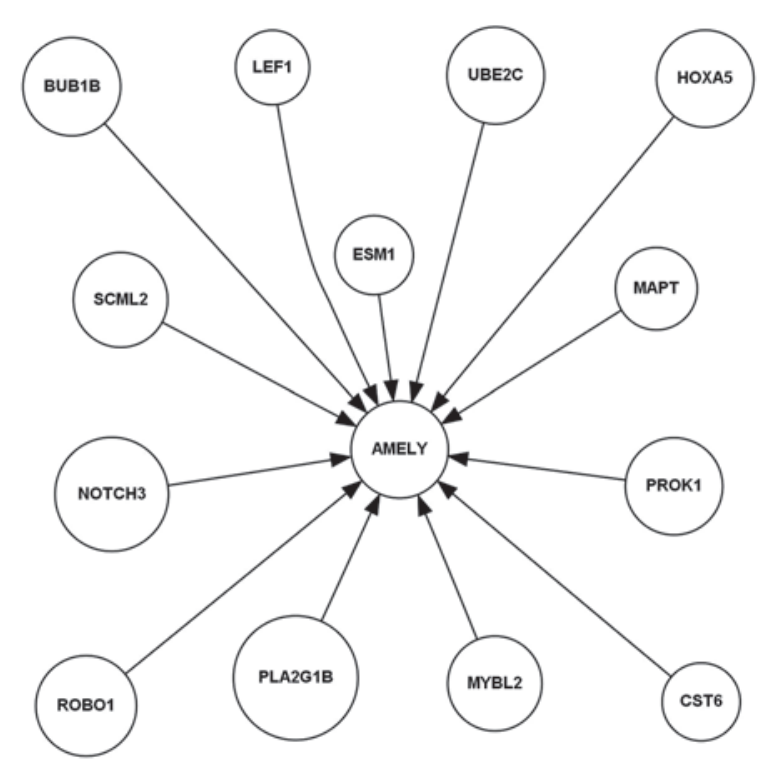

Figure 2. Amelogenin Y-linked (AMELY)-activated upstream regulation molecular network in human hepatocellular carcinoma (HCC) by GRNInfer and our programming.
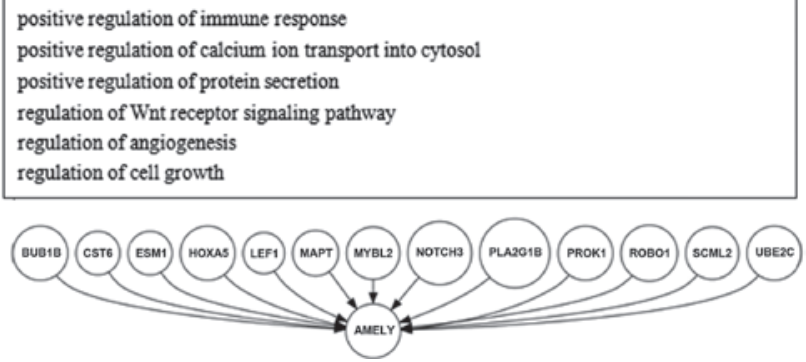

Figure 3. A model of the activated amelogenin Y-linked (AMELY) upstream regulation molecular network in human hepatocellular carcinoma (HCC) from different comparisons with the corresponding activated gene ontology (GO) molecular network of non-tumor hepatitis/cirrhotic tissues.

of calcium ion transport into the cytosol, cell proliferation, DNA replication, fibroblast proliferation, immune response, microtubule polymerization, protein secretion, and specific transcription from RNA polymerase II promoter. There was also regulation of angiogenesis, cell growth, protein metabolism and the Wnt receptor signaling pathway. The processes common to both included positive regulation of transcription. It was therefore postulated that $A M E L Y$-activated coupling upstream positively regulates the immune response-mediated protein secretion to Wnt signaling and calcium into cytosolinduced regulation of cell growth and angiogenesis in HCC.

The correlations between regulation of immune response, protein secretion, Wnt signaling and calcium movement into the cytosol with regulation of cell growth or angiogenesis have been reported. Examples include calcium store sensor stromal-interaction molecule 1-dependent signaling playing an important role in cervical cancer growth, migration and angiogenesis (37); blockade of Wnt signaling inhibiting angiogenesis and tumor growth in HCC (38); enhancement of the recognized Wnt/ $\beta$-catenin signaling activity by $\mathrm{HCV}$ core protein promoting cell growth of HCC cells (39); porous membrane substrates offering better niches to enhance the Wnt 
signaling and promote human embryonic stem cell growth and differentiation (40); effect of dietary tea polyphenols on growth performance and cell-mediated immune response of post-weaning piglets under oxidative stress (41); Wnt inhibitory factor 1 inducing apoptosis and inhibiting cervical cancer growth, invasion and angiogenesis in vivo (42); Rspol/Wnt signaling promoting angiogenesis via Vegfc/Vegfr3 (43); bone morphogenetic protein 2 inducing pulmonary angiogenesis via Wnt- $\beta$-catenin and Wnt-RhoA-Racl pathways (44); and the correlation between angiogenesis and the immune response in carcinogenesis and the progression of malignant disease (45).

AMELY-activated upstream regulation molecular networks in non-tumor hepatitis/cirrhotic tissues and HCC were constructed (Figs. 1 and 2). We further constructed a model of the AMELY upstream regulation molecular network in HCC only, which included BUB1B, CST6, ESM1, HOXA5, LEF1, MAPT, MYBL2, NOTCH3, PLA2G1B, PROK1, ROBO1, $S C M L 2$ and $U B E 2 C$, by comparison with the corresponding activated GO molecular network of non-tumor hepatitis/ cirrhotic tissues (Fig. 3).

In summary, the biological processes and occurrence numbers of GO in HCC tissue with high expression (fold change $\geq 2$ ) of $A M E L Y$-activated upstream regulation network were compared with those of the corresponding non-tumor hepatitis/ cirrhotic tissues with low expression of the activated network. The biological processes in the non-tumor hepatitis/cirrhotic tissues included positive regulation of mismatch repair, regulation of transcription from RNA polymerase II promoter, negative regulation of cell-cell adhesion, protein ubiquitination and protein catabolism. The processes in HCC consisted of positive regulation of calcium ion transport into the cytosol, cell proliferation, DNA replication, fibroblast proliferation, immune response, microtubule polymerization, protein secretion and specific transcription from RNA polymerase II promoter; regulation of angiogenesis, cell growth, protein metabolism and the Wnt receptor signaling pathway; and negative regulation of endothelial cell differentiation, microtubule depolymerization, peptidase activity and progression through the cell cycle. A common factor included positive regulation of transcription. Activated AMELY coupling upstream positive regulation of immune response-mediated protein secretion to Wnt signaling and calcium into cytosol-induced regulation of cell growth and angiogenesis network in HCC was proposed. The $A M E L Y$ upstream regulation molecular network model was constructed, including BUB1B, CST6, ESM1, HOXA5, LEF1, MAPT, MYBL2, NOTCH3, PLA2G1B, PROK1, $R O B O 1, S C M L 2$ and $U B E 2 C$, in HCC from a GEO dataset by gene regulation network inference methods and computational programming.

\section{Acknowledgements}

This study was supported by the National Natural Science Foundation of China (No. 61171114), the Returned Overseas Chinese Scholars for Scientific Research Foundation of State Education Ministry, the Significant Science and Technology Project for New Transgenic Biological Species (2009ZX08012001B), Automatical Scientific Planning of Tsinghua University (20111081023 and 20111081010) and the State Key Laboratory of Pattern Recognition Open Foundation.

\section{References}

1. Hoshida Y, Villanueva A, Kobayashi M, et al: Gene expression in fixed tissues and outcome in hepatocellular carcinoma. N Engl J Med 359: 1995-2004, 2008

2. Veenbergen $S$ and van Spriel AB: Tetraspanins in the immune response against cancer. Immunol Lett 138: 129-136, 2011.

3. Nishikawa M, Otsuki T, Ota A, et al: Induction of tumor-specific immune response by gene transfer of Hsp70-cell-penetrating peptide fusion protein to tumors in mice. Mol Ther 18: 421-428, 2010.

4. Ladoire S, Mignot G, Dabakuyo S, et al: In situ immune response after neoadjuvant chemotherapy for breast cancer predicts survival. J Pathol 224: 389-400, 2011.

5. Fucikova J, Kralikova P, Fialova A, et al: Human tumor cells killed by anthracyclines induce a tumor-specific immune response. Cancer Res 71: 4821-4833, 2011.

6. Depianto D, Kerns ML, Dlugosz AA and Coulombe PA: Keratin 17 promotes epithelial proliferation and tumor growth by polarizing the immune response in skin. Nat Genet 42: 910-914, 2010.

7. Cunha LL, Tincani AJ, Assumpcao LV, Soares FA, Vassallo J and Ward LS: Interleukin-10 but not interleukin-18 may be associated with the immune response against well-differentiated thyroid cancer. Clinics (Sao Paulo) 66: 1203-1208, 2011.

8. Zabel BA, Miao Z, Lai NL, et al: CXCR7 protein expression correlates with elevated mmp-3 secretion in breast cancer cells. Oncol Lett 1: 845-847, 2010.

9. Nirde P, Derocq D, Maynadier M, et al: Heat shock cognate 70 protein secretion as a new growth arrest signal for cancer cells. Oncogene 29: 117-127, 2010.

10. Milewicz T, Rýs J, Wójtowicz A, et al: Overexpression of P53 protein and local hGH, IGF-I, IGFBP-3, IGFBP-2 and PRL secretion by human breast cancer explants. Neuro Endocrinol Lett 32: 328-333, 2011.

11. Lim JW, Mathias RA, Kapp EA, et al: Restoration of full-length APC protein in SW480 colon cancer cells induces exosomemediated secretion of DKK-4. Electrophoresis 33: 1873-1880, 2012.

12. Jube S, Rivera ZS, Bianchi ME, et al: Cancer cell secretion of the DAMP protein HMGB1 supports progression in malignant mesothelioma. Cancer Res 72: 3290-3301, 2012.

13. de Sousa EM, Vermeulen L, Richel D and Medema JP: Targeting Wnt signaling in colon cancer stem cells. Clin Cancer Res 17: 647-653, 2011 .

14. Corbo C, Orru S, Gemei M, et al: Protein cross-talk in CD133+ colon cancer cells indicates activation of the Wnt pathway and upregulation of SRp20 that is potentially involved in tumorigenicity. Proteomics 12: 2045-2059, 2012.

15. Cha YH, Kim NH, Park C, Lee I, Kim HS and Yook JI: MiRNA-34 intrinsically links p53 tumor suppressor and Wnt signaling. Cell Cycle 11: 1273-1281, 2012.

16. Cai $\mathrm{C}$ and Zhu X: The Wnt/ $\beta$-catenin pathway regulates selfrenewal of cancer stem-like cells in human gastric cancer. Mol Med Rep 5: 1191-1196, 2012.

17. Boparai KS, Dekker E, Polak MM, Musler AR, van Eeden S and van Noesel CJ: A serrated colorectal cancer pathway predominates over the classic WNT pathway in patients with hyperplastic polyposis syndrome. Am J Pathol 178: 2700-2707, 2012.

18. Andrade Filho PA, Letra A, Cramer A, et al: Insights from studies with oral cleft genes suggest associations between WNT-pathway genes and risk of oral cancer. J Dent Res 90: 740-746, 2011.

19. Sitdikova GF, Weiger TM and Hermann A: Hydrogen sulfide increases calcium-activated potassium (BK) channel activity of rat pituitary tumor cells. Pflugers Arch 459: 389-397, 2010.

20. Storey JD: A direct approach to false discovery rates. J Roy Stat Soc, Ser B 64: 479-498, 2002.

21. Wang Y, Joshi T, Zhang XS, Xu D and Chen L: Inferring gene regulatory networks from multiple microarray datasets. Bioinformatics 22: 2413-2420, 2006.

22. Huang J, Wang L, Jiang $M$ and Zheng $X$ : Interferon $\alpha$-inducible protein 27 computational network construction and comparison between the frontal cortex of HIV encephalitis (HIVE) and HIVE-control patients. Open Genomics J 3: 1-8, 2010.

23. Huang JX, Wang L and Jiang MH: TNFRSF11B computational development network construction and analysis between frontal cortex of HIV encephalitis (HIVE) and HIVE-control patients. J Inflamm (Lond) 7: 50, 2010. 
24. Sun L, Wang L, Jiang M, Huang J and Lin H: Glycogen debranching enzyme 6 (AGL), enolase 1 (ENOSF1), ectonucleotide pyrophosphatase 2(ENPP2 1), glutathione S-transferase 3 (GSTM3_3) and mannosidase (MAN2B2) metabolism computational network analysis between chimpanzee and human left cerebrum. Cell Biochem Biophys 61: 493-505, 2011.

25. Sun Y, Wang L, Jiang M, Huang J, Liu Z and Wolfl S: Secreted phosphoprotein 1 upstream invasive network construction and analysis of lung adenocarcinoma compared with human normal adjacent tissues by integrative biocomputation. Cell Biochem Biophys 56: 59-71, 2010.

26. Sun Y, Wang L and Lui L: Integrative decomposition procedure and Kappa statistics set up ATF2 ion binding module in Malignant Pleural Mesothelioma (MPM). Frontiers of Electrical and Electronic Engineering in China 3: 381-387, 2008.

27. Wang L, Huang J and Jiang M: CREB5 computational regulation network construction and analysis between frontal cortex of HIV encephalitis (HIVE) and HIVE-control patients. Cell Biochem Biophys 60: 199-207, 2011.

28. Wang L, Huang J and Jiang M: RRM2 computational phosphoprotein network construction and analysis between no-tumor hepatitis/cirrhotic liver tissues and human hepatocellular carcinoma (HCC). Cell Physiol Biochem 26: 303-310, 2011.

29. Wang L, Huang J, Jiang M and Lin H: Tissue-specific transplantation antigen P35B (TSTA3) immune response-mediated metabolism coupling cell cycle to postreplication repair network in no-tumor hepatitis/cirrhotic tissues (HBV or HCV infection) by biocomputation. Immunol Res 52: 258-268, 2012.

30. Wang L, Huang J, Jiang M and Lin H: Signal transducer and activator of transcription 2 (STAT2) metabolism coupling postmitotic outgrowth to visual and sound perception network in human left cerebrum by biocomputation. J Mol Neurosci 47: 649-658, 2012.

31. Wang L, Huang J, Jiang M and Sun L: Survivin (BIRC5) cell cycle computational network in human no-tumor hepatitis/ cirrhosis and hepatocellular carcinoma transformation. J Cell Biochem 112: 1286-1294, 2011.

32. Wang L, Huang J, Jiang M and Sun L: MYBPC1 computational phosphoprotein network construction and analysis between frontal cortex of HIV encephalitis (HIVE) and HIVE-control patients. Cell Mol Neurobiol 31: 233-241, 2011.

33. Wang L, Huang J, Jiang M and Zheng X: AFP computational secreted network construction and analysis between human hepatocellular carcinoma (HCC) and no-tumor hepatitis/cirrhotic liver tissues. Tumour Biol 31: 417-425, 2010.
34. Wang L, Sun L, Huang J and Jiang M: Cyclin-dependent kinase inhibitor 3 (CDKN3) novel cell cycle computational network between human non-malignancy associated hepatitis/cirrhosis and hepatocellular carcinoma (HCC) transformation. Cell Prolif 44: 291-299, 2011.

35. Gao P, Sun X, Chen X, Subjeck J and Wang XY: Secretion of stress protein grp170 promotes immune-mediated inhibition of murine prostate tumor. Cancer Immunol Immunother 58: 1319-1328, 2009.

36. Wang L, Sun Y, Jiang M and Zheng X: Integrative decomposition procedure and Kappa statistics for the distinguished single molecular network construction and analysis. J Biomed Biotechnol 2009: 726728, 2009.

37. Chen YF, Chiu WT, Chen YT, et al: Calcium store sensor stromalinteraction molecule 1-dependent signaling plays an important role in cervical cancer growth, migration, and angiogenesis. Proc Natl Acad Sci USA 108: 15225-15230, 2011.

38. Hu J, Dong A, Fernandez-Ruiz V, et al: Blockade of Wnt signaling inhibits angiogenesis and tumor growth in hepatocellular carcinoma. Cancer Res 69: 6951-6959, 2009.

39. Liu J, Ding X, Tang J, et al: Enhancement of canonical Wnt/ßcatenin signaling activity by $\mathrm{HCV}$ core protein promotes cell growth of hepatocellular carcinoma cells. PLoS One 6: e27496, 2011.

40. Jin S, Yao H, Krisanarungson P, Haukas A and Ye K: Porous membrane substrates offer better niches to enhance the wnt signaling and promote human embryonic stem cell growth and differentiation. Tissue Eng Part A 18: 1419-1430, 2012.

41. Deng Q, Xu J, Yu B, et al: Effect of dietary tea polyphenols on growth performance and cell-mediated immune response of post-weaning piglets under oxidative stress. Arch Anim Nutr 64: $12-21,2010$

42. Ramachandran I, Thavathiru E, Ramalingam S, et al: Wnt inhibitory factor 1 induces apoptosis and inhibits cervical cancer growth, invasion and angiogenesis in vivo. Oncogene 31: 2725-2737, 2012.

43. Gore AV, Swift MR, Cha YR, et al: Rspo1/Wnt signaling promotes angiogenesis via Vegfc/Vegfr3. Development 138: 4875-4886, 2011.

44. de Jesus Perez VA, Alastalo TP, Wu JC, et al: Bone morphogenetic protein 2 induces pulmonary angiogenesis via Wnt- $\beta$-catenin and Wnt-RhoA-Rac1 pathways. J Cell Biol 184: 83-99, 2009.

45. O'Byrne KJ, Dalgleish AG, Browning MJ, Steward WP and Harris AL: The relationship between angiogenesis and the immune response in carcinogenesis and the progression of malignant disease. Eur J Cancer 36: 151-169, 2000. 